\title{
Magnetic and Structural Properties of EuS-PbS Multilayers Grown on $n$-PbS (100) Substrates
}

M. Chernyshova ${ }^{a}$, E. Lusakowska ${ }^{a}$, V. Domukhovski ${ }^{a}$, K. Grasza ${ }^{a}$, A. Szczerbakow ${ }^{a}$, S. WroteK ${ }^{a}$, L. KowalczYK ${ }^{a}$, T. StorY ${ }^{a}$, C.J.P. Smits ${ }^{b}$, H.J.M. SwaGteN ${ }^{b}$, W.J.M. De JongE ${ }^{b}$, W. Palosz ${ }^{c}$, A.Yu. Sipatov ${ }^{d}$ AND V.V. VOLOBUEV ${ }^{d}$

${ }^{a}$ Institute of Physics, Polish Academy of Sciences al. Lotników 32/46, 02-668 Warsaw, Poland

${ }^{b}$ Department of Applied Physics, Center for NanoMaterials and COBRA Research Institute, Eindhoven University of Technology P.O. Box 513, 5600 MB Eindhoven, The Netherlands ${ }^{c}$ USRA-NASA/Marshal Space Flight Center, Huntsville, Alabama 35812, USA ${ }^{d}$ National Technical University KPI, 21 Frunze Str., 61002 Kharkov, Ukraine

\footnotetext{
Magnetic properties of semiconductor $\operatorname{EuS}(t)-\mathrm{PbS}(d)-\operatorname{EuS}(t)$ ferromagnetic trilayers $(t=30 \div 300 \AA$ and $d=7.5 \div 70 \AA$ ) grown on $n$-type monocrystalline $\mathrm{PbS}(100)$ substrate were studied by SQUID magnetometry and ferromagnetic resonance technique yielding, in particular, the dependence of the ferromagnetic Curie temperature on the thickness of the EuS layer. Structural parameters of layers were examined by X-ray powder diffraction analysis. A high structural quality of the substrate and the multilayer was verified by the measurements of the X-ray rocking curve width indicating the values of the order of 100 arcsec and by atomic force microscopy revealing the presence on the cleft $\mathrm{PbS}$ surface regions practically flat in the atomic scale over the area of $1 \times 0.1 \mu \mathrm{m}^{2}$.
}

PACS numbers: $75.20 . \mathrm{Ck}, 75.30$.Et 


\section{Introduction}

In EuS-PbS semiconductor multilayers ferromagnetic layers of EuS form electronic barriers for nonmagnetic PbS quantum wells. Both materials crystallize in a cubic (rocksalt) structure and are well matched with respect to their lattice parameters: for EuS $a_{0}=5.97 \AA$ whereas for PbS $a_{0}=5.94 \AA$. Therefore, epitaxial EuS-PbS multilayers can be grown pseudomorphically up to the overall thickness of the structure exceeding $2000 \AA$. Until now the EuS-PbS multilayers were grown on two insulating, transparent in the near infrared and in the visible ranges of spectrum monocrystalline substrates: $\mathrm{BaF}_{2}$ with growth proceeding along (111) crystal axis and $\mathrm{KCl}$ with growth along (100) direction. In both cases the layers were deposited on freshly cleaved surfaces. Ferromagnetic transition in EuS-PbS/ $\mathrm{BaF}_{2}$ and EuS-PbS/KCl multilayers is observed even in structures with ultrathin layers of EuS of only $6 \AA$, i.e. two monolayers (2 ML). Ferromagnetic transition temperature $T_{\mathrm{c}}$ depends on the thickness $t$ of the ferromagnetic layer of EuS and on the strain introduced by the difference in the thermal expansion coefficients of the substrate and the multilayer [1,2]. Particularly interesting are $\mathrm{EuS}-\mathrm{PbS}$ multilayers grown on $\mathrm{KCl}$ (100) substrates in which, for very thin $\mathrm{PbS}$ spacer layers, the antiferromagnetic interlayer coupling is observed in neutron diffraction and magnetization experiments $[3,4]$. The ferromagnetic mutual orientation of magnetization vectors of EuS layers can be obtained by the application of small external magnetic fields of 100 Oe.

In this work, we examine the magnetic and structural properties of EuS-PbS multilayers grown on $n$-type $\mathrm{PbS}(100)$ substrates cleaved from the bulk single crystals prepared by various modifications of physical vapor transport method. The development of conducting $n$-type (100)-oriented monocrystalline $\mathrm{PbS}$ substrates for the epitaxial growth of EuS-PbS multilayers qualitatively expands our possibilities aiming at the development of new semiconductor spintronic tunneling and light emitting structures actively exploiting ferromagnetic character of EuS barriers for the control of the charge and spin transport perpendicular to the plane of the structure.

\section{Growth and characterization}

For the growth of EuS-PbS multilayers studied in this work we used $\mathrm{PbS}$ (100) substrates freshly cleaved from bulk PbS crystals. The bulk PbS monocrystals were grown by two modifications of physical vapor transport method: the method of self-selecting vapor growth (SSVG, [5]), and the low super-saturation "contactless" physical vapor transport (LSS PVT, [6]) method. The application of both methods resulted in a successful growth of high quality bulk PbS monocrystals with (100) crystal cleavage planes. From single-crystalline blocks of $\mathrm{PbS}$ (with their volume ranging from a half to a few cubic centimeters) we selected for the epitaxial deposition of EuS-PbS multilayers a number of freshly cleaved 
(100)-oriented plates about $1 \mathrm{~mm}$ thick with a typical area of about $4 \times 8 \mathrm{~mm}^{2}$. The X-ray powder diffraction analysis (Cu $K_{\alpha}$ radiation, see Fig. 1) of PbS bulk crystals showed, as expected, the rocksalt structure with the lattice parameter $a_{0}=5.938 \AA$ for crystals grown by SSVG method and $a_{0}=5.937 \AA$ for crystals grown by LSS PVT method. To characterize the crystal quality of these PbS plates we measured the width of the X-ray rocking curve for the (200) reflex. It shows the FWHM values in the range of 65-250 arcsec (see inset in Fig. 1) for the set of about ten $\mathrm{PbS}$ substrates studied by us. The atomic force microscopy (AFM)

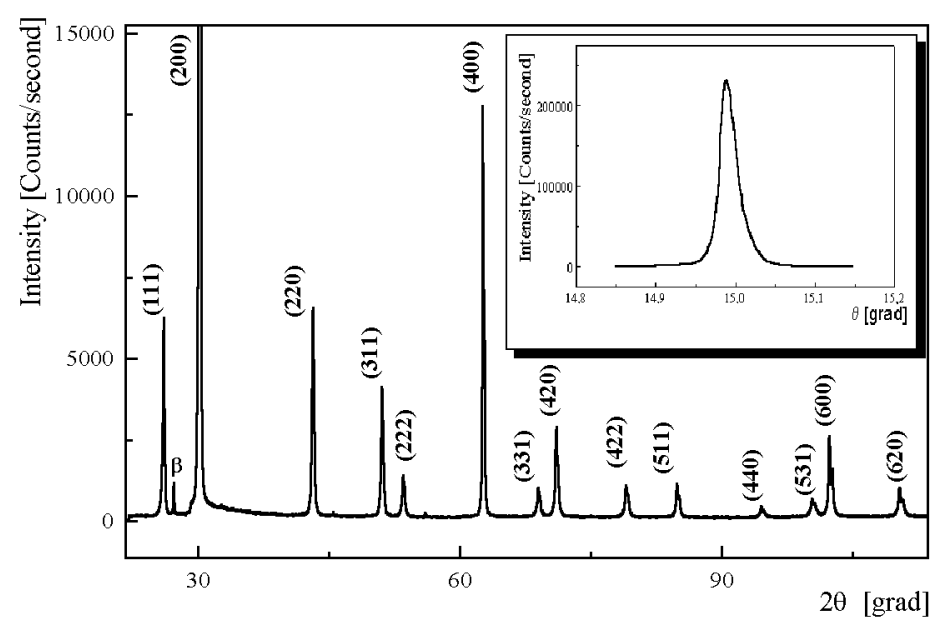

Fig. 1. The X-ray powder diffraction spectrum for PbS substrate revealing rocksalt lattice with $a_{0}=5.937 \AA$. The inset shows the rocking curve for $(200)$ reflex for $\mathrm{PbS}$ monocrystal.

analysis of $\mathrm{PbS}$ substrate plates indicated the root-mean-square (rms) roughness of $10 \AA$, i.e., about $3 \mathrm{ML}$ for the analyzed area of $10 \times 10 \mu \mathrm{m}^{2}$. The characteristic feature of surface morphology of $\mathrm{PbS}$ substrate plates is the existence of very long (in some cases exceeding $10 \mu \mathrm{m}$ ) practically atomically flat regions with a typical area of $1 \times 0.1 \mu \mathrm{m}^{2}$. Both techniques of PVT growth applied in this work to the growth of $\mathrm{PbS}$ produced $n$-type material as checked by Hall effect and thermoelectric measurements. It is the consequence of the composition of the source polycrystalline $\mathrm{PbS}$ material which is usually intentionally prepared with a slight deviation from stoichiometric composition towards excess metal (deficient sulfur). In all IV-VI semiconductor compounds the anion vacancies are the well-known source of high concentration (typically $n \simeq 10^{17} \div 10^{18} \mathrm{~cm}^{-3}$ ) of quasi-free electrons. As the electronic energy levels of these defects are located above the bottom of the conduction band one observes metallic $n$-type conductivity with practically temperature independent electron concentration. 
The EuS-PbS multilayers were grown on PbS(100) substrates employing vacuum deposition system equipped with an electron gun for the evaporation of EuS and tungsten boats for standard resistive heating of PbS. The thickness of the layers was controlled in-situ by quartz resonator. The substrate temperature during the growth was about $350^{\circ} \mathrm{C}$. As the lattice parameters of $\mathrm{PbS}$ and EuS are very similar the X-ray diffraction analysis of EuS-PbS/PbS(100) structures shows only a single set of diffraction peaks corresponding very closely to the rocksalt lattice parameter of $\mathrm{PbS}$. This is in contrast to $\mathrm{EuS}-\mathrm{PbS} / \mathrm{BaF}_{2}$ and $\mathrm{EuS}-\mathrm{PbS} / \mathrm{KCl}$ structures in which the substantial mismatch between the substrate and the layers is reflected in X-ray measurements as separate sets of diffraction peaks. The FWHM rocking curve width for EuS-PbS/PbS structures (typically 200 arcsec) was somewhat larger than for the freshly cleft $\mathrm{PbS}$ crystals but compares favorably even with the best structures grown previously on $\mathrm{BaF}_{2}$ or $\mathrm{KCl}$ substrates for which the rocking curve width of 300 arcsec was found. The AFM analysis of the morphology of the top PbS layer in the EuS-PbS multilayer showed rms roughness of $40 \AA$ for the analyzed region of $10 \times 10 \mu \mathrm{m}^{2}$. The measurements were taken in the air with no special cleaning procedure. Our measurements indicate that the morphology of the top layer is influenced by a slow oxidation of PbS layer. Therefore, most of our structures were capped with $100-1000 \AA$ PbS protective layer. In structures intended for transport measurements capping of the top PbS layer with metallic $\mathrm{Au}$ or and $\mathrm{LaB}_{6}$ ohmic contact layers is also frequently employed.

\section{Magnetic properties}

Magnetic properties of EuS-PbS/PbS(100) multilayers were studied by a superconducting quantum interference (SQUID) device for magnetization measurements as a function of temperature $(T=5 \div 35 \mathrm{~K})$ and magnetic field $(B \leq 5 \mathrm{~T})$ as well as by the ferromagnetic resonance (FMR) technique. We studied $\operatorname{EuS}(t)-\operatorname{PbS}(d)-\operatorname{EuS}(t)$ ferromagnetic trilayers with the thickness of ferromagnetic EuS layers $t=30 \div 300 \AA$ and the thickness of nonmagnetic PbS spacer $d=7.5 \div 70 \AA$. The EuS-PbS-EuS trilayers were deposited on PbS substrate overgrown with a homoepitaxial $450 \AA$ thick PbS buffer layer. The entire EuS-PbS trilayer structure was capped with a $500 \AA$ top PbS layer.

Ferromagnetic transition is observed in all EuS-PbS/PbS structures studied by us. The temperature dependence of magnetization as well as the magnetic hysteresis loop of the reference $\mathrm{PbS}-\mathrm{EuS}-\mathrm{PbS} / \mathrm{PbS}(100)$ structure with a single thick (300 $\AA$ ) EuS layer is presented in Fig. 2. The ferromagnetic Curie temperature $T_{c}$ of EuS-PbS/PbS multilayers decreases from the value $17.0 \mathrm{~K}$ observed for the multilayer with a $300 \AA$ thick EuS layer to $15.0 \mathrm{~K}$ for trilayers with $30 \AA$ EuS layers. The dependence of the transition temperature on the thickness of EuS layer is presented in Fig. 3 for all three substrates used for epitaxial deposition of EuS layers. The $T_{\mathrm{c}}(t)$ dependence for $\mathrm{EuS}-\mathrm{PbS} / \mathrm{PbS}(100)$ multilayers is analogous 

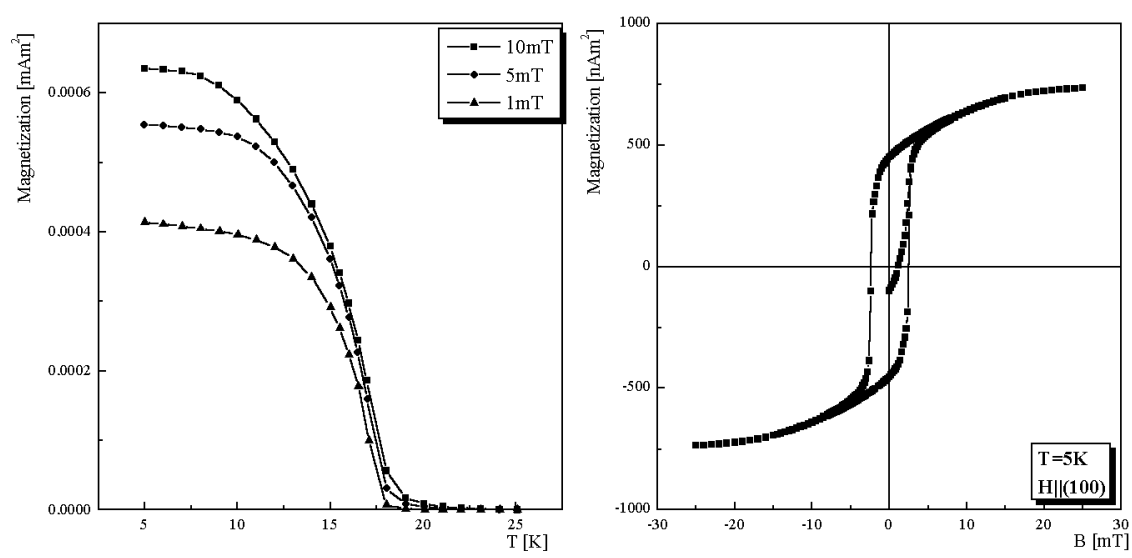

Fig. 2. Ferromagnetic transition $\left(T_{c}=17.0 \mathrm{~K}\right)$ in $\operatorname{PbS}(100 \AA)-\operatorname{EuS}(300 \AA)-\operatorname{PbS}(100 \AA)$ $/ \mathrm{PbS}(100)$ structure with a single thick layer of $\mathrm{EuS}$; (a) the temperature dependence of magnetization; (b) the magnetic hysteresis loop.

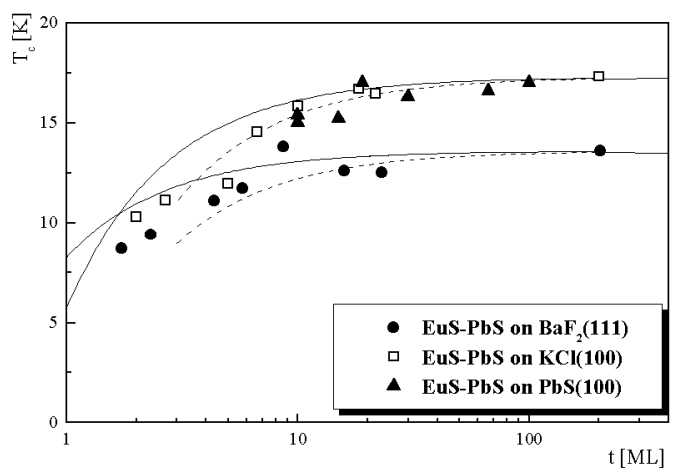

Fig. 3. The dependence of the ferromagnetic Curie temperature of EuS-PbS multilayers grown on various substrates on the thickness of EuS layer. The solid lines indicate the mean-field theoretical model with sharp interfaces whereas the broken lines correspond to the mean field model for $\pm 1 \mathrm{ML}$ interdiffused interface [1].

to the case of EuS-PbS structures grown on $\mathrm{KCl}(100)$ substrates and can be accounted for both by a simple mean-field model taking into account the distribution of magnetic neighbors at the magnetic-nonmagnetic interface [1] as well as by more rigorous theoretical models employing Green function techniques for the analysis of magnon excitations in these multilayers [7]. The substantial (by the factor 2/3) reduction of the Curie temperature is expected only for EuS layers thinner than about $10 \mathrm{ML}$ as experimentally observed in $\mathrm{EuS}-\mathrm{PbS} / \mathrm{KCl}$ and $\mathrm{EuS}-\mathrm{PbS} / \mathrm{BaF}_{2}$ structures (see Fig. 3). It is interesting to note that the ferromagnetic Curie tem- 
perature $T_{\mathrm{c}}=17.0 \mathrm{~K}$ observed in the structures with thick EuS layers grown on $\mathrm{PbS}$ substrate is by about $0.4 \mathrm{~K}$ higher than the transition temperature in the bulk EuS crystals. This result can be understood taking into account that EuS- $\mathrm{PbS} / \mathrm{PbS}$ multilayers are expected to be pseudomorphically strained with the EuS layer being biaxially compressed. It results in the decrease in the distance between nearest magnetic neighbors and the increase in the relevant exchange integrals. This effect is discussed in detail in Ref. [1] in which the large strain-induced shift of Curie temperature was experimentally observed in EuS-PbS/KCl structures as a consequence of both EuS/PbS lattice mismatch and the difference of thermal expansion coefficients between the $\mathrm{KCl}$ substrate and the multilayer (also resulting in compressive strain in EuS layer).

Ferromagnetic transition temperatures in EuS-PbS/PbS structures were also clearly observed in X-band magnetic resonance measurements carried out over the temperature range of $T=3.5 \div 60 \mathrm{~K}$. For this work, an important piece of information obtained from the FMR measurements concerns the width of the FMR line. In EuS-PbS/PbS(100) multilayers the FMR line width is about 100-150 Oe as compared to 300-400 Oe observed in EuS-PbS/KCl structures and 400-600 Oe found in $\mathrm{EuS}-\mathrm{PbS} / \mathrm{BaF}_{2}$ multilayers. It supports our conclusions of a better quality of EuS-PbS multilayers grown on PbS (100) as compared to the layers grown on other substrates.

We have also examined the magnetic properties of EuS-PbS-EuS/PbS trilayers with a very thin nonmagnetic $\mathrm{PbS}$ spacer. For a number of multilayers with PbS thickness $d=7.5 \AA$ and $d=10 \AA$ we observed pronounced magnetic effects (e.g. very low magnetic remanence, characteristic kink on magnetic hysteresis loops and non-monotonic, at low fields, temperature dependence of magnetization) which reveal the presence of antiferromagnetic interlayer coupling between EuS layers. This finding is important for the development of new spintronic structures as it offers the possibility to switch between antiferromagnetic and ferromagnetic alignment of the magnetization vectors of EuS layers applying weak external magnetic fields of 100 Oe. This effect is discussed in detail in Ref. [8].

\section{Summary}

A successful application of physical vapor transport method for the growth of $\mathrm{PbS}$ crystals allows us to obtain a high structural quality of single-crystal materials with (100)-oriented cleaved surfaces exhibiting morphological perfection suitable for the epitaxial growth of EuS-PbS ferromagnetic multilayers. Magnetization and magnetic resonance studies show that the Curie temperature of the ferromagnetic transition in EuS-PbS-EuS/PbS(100) trilayers decreases with decreasing thickness of the magnetic layer. For a thick single layer of EuS grown on $\mathrm{PbS}$ the Curie temperature is $17.0 \mathrm{~K}$, i.e. about $0.4 \mathrm{~K}$ above the transition temperature in the bulk EuS crystals. This effect is likely to arise from the biaxial compressing strain 
expected in pseudomorphic EuS-PbS structures due to the difference in lattice parameters of the two layers. For structures with ultrathin $\mathrm{PbS}$ spacers the magnetic hysteresis loops and as well as the characteristic temperature dependence of magnetization reveal the presence of antiferromagnetic interlayer coupling.

\section{Acknowledgments}

This work was partially supported by the State Committee for Scientific Research project No. 2 P03B 15418 and within European Community program ICA1-CT-2000-70018 (Center of Excellence CELDIS). Partial support of the office of Biological and Physical Sciences of NASA is greatly appreciated.

\section{References}

[1] A. Stachow-Wójcik, T. Story, W. Dobrowolski, M. Arciszewska, R.R. Gałązka, M.W. Kreijveld, C.H.W. Swüste, H.J.M. Swagten, W.J.M. de Jonge, A. Twardowski, A.Yu. Sipatov, Phys. Rev. B 60, 15220 (1999).

[2] L. Kowalczyk, J. Sadowski, R.R. Gałązka, A. Stachow-Wójcik, A.Yu. Sipatov, V.A. Smirnov, Acta Phys. Pol. A 94, 357 (1998).

[3] H. Kępa, J. Kutner-Pielaszek, J. Blinowski, A. Twardowski, C.F. Majkrzak, T. Story, P. Kacman, R.R. Gałązka, K. Ha, H.J.M. Swagten, W.J.M. de Jonge, A.Yu. Sipatov, V.V. Volobuev, T.M. Giebultowicz, Europhys. Lett. 56, 54 (2001).

[4] L. Kowalczyk, M. Chernyshova, T. Story, J.K. Ha, A.Yu. Sipatov, V.V. Volobuev, Acta Phys. Pol. A 100, 357 (2001).

[5] A. Szczerbakow, Crystal Growth and Design 1, 183 (2001).

[6] K. Grasza, W. Palosz, Cryst. Res. Technol. 34, 565 (1999).

[7] R. Świrkowicz, W. Mac, A. Twardowski, T. Story, A.Yu. Sipatov, to be published.

[8] M. Chernyshova, L. Kowalczyk, A. Szczerbakow, T. Story, C.J.P. Smits, H.J.M. Swagten, J.K. Ha, W.J.M. de Jonge, A.Yu. Sipatov, V.V. Volobuev, in: Proc. Int. Conf. Physics and Applications of Spin Related Phenomena in Semiconductors, Würzburg 2002, Ed. L.W. Molenkamp, to be published in J. Supercond. 